\title{
Weak, strong and detached oblique shocks in gravity-driven granular free-surface flows
}

\author{
J. M. N. T. GRAY AND X. CUI \\ School of Mathematics and Manchester Centre for Nonlinear Dynamics, University of Manchester, \\ Manchester M13 9PL, UK
}

(Received 8 March 2006 and in revised form 15 November 2006)

Hazardous natural flows such as snow-slab avalanches, debris flows, pyroclastic flows and lahars are part of a much wider class of dense gravity-driven granular freesurface flows that frequently occur in industrial processes as well as in foodstuffs in our kitchens! This paper investigates the formation of oblique granular shocks, when the oncoming flow is deflected by a wall or obstacle in such a way as to cause a rapid change in the flow height and velocity. The theory for non-accelerative slopes is qualitatively similar to that of gasdynamics. For a given deflection angle there are three possibilities: a weak shock may form close to the wall; a strong shock may extend across the chute; or the shock may detach from the tip. Weak shocks have been observed in both dense granular free-surface flows and granular gases. This paper shows how strong shocks can be triggered in chute experiments by careful control of the downstream boundary conditions. The resulting downstream flow height is much thicker than that of weak shocks and there is a marked decrease in the downstream velocity. Strong shocks therefore dissipate much more energy than weak shocks. An exact solution for the angle at which the flow detaches from the wedge is derived and this is shown to be in excellent agreement with experiment. It therefore provides a very useful criterion for determining whether the flow will detach. In experimental, industrial and geophysical flows the avalanche is usually accelerated, or decelerated, by the net effect of the gravitational acceleration and basal sliding friction as the slope inclination angle changes. The presence of these source terms necessarily leads to gradual changes in the flow height and velocity away from the shocks, and this in turn modifies the local Froude number of the flow. A shock-capturing non-oscillating central method is used to compute numerical solutions to the full problem. This shows that the experiments can be matched very closely when the source terms are included and explains the deviations away from the classical oblique-shock theory. We show that weak shocks bend towards the wedge on accelerative slopes and away from it on decelerative slopes. In both cases the presence of the source terms leads to a gradual increase in the downstream flow thickness along the wedge, which suggests that defensive dams should increase in height further down the slope, contrary to current design criteria but in accordance with field observations of snow-avalanche deposits from a defensive dam in Northwestern Iceland. Movies are available with the online version of the paper.

\section{Introduction}

Many geophysical and industrial granular flows occur as dense free-surface avalanches that are driven downslope under the action of gravity. In geophysics these 
encompass a range of large-scale natural hazards including snow-slab avalanches (Savage \& Hutter 1989), dense pyroclastic flows (Sparks et al. 2002), debris flows (Iverson 1997) and lahars (Vallance 2000), which are a serious threat to many populations living in the vicinity of mountains and volcanoes. Granular avalanches also occur in smaller-scale industrial processes in the bulk-chemical, mining and pharmaceutical industries. They are found in silos, stockpiles, rotating mixers (Gray 2001) and continuous-feed inclined rotating kilns (Spurling, Davidson \& Scott 2001), used to store, mix or process grains. Shock waves frequently occur in the free-surface layers and, in combination with the interaction between the surface and the underlying solid body of grains, leads to novel segregation and mixing effects that are still poorly understood (Shinbrot \& Muzzio 2000).

An interesting feature of granular avalanches is that they exhibit a weak scale dependence for volumes below $10^{6} \mathrm{~m}^{3}$. Small-scale laboratory experiments can therefore be used to yield important insight into larger-scale geophysical and industrial flows. Continuum theories reflect this weak scale dependence and are often constructed to be scale-invariant. The first models were developed in Russia in the 1960s and 70s (Grigorian, Eglit \& Iakimov 1967; Kulikovskii \& Eglit 1973) to calculate the run-out distance and the associated risk posed by snow-slab avalanches in the Urals. These models were formulated by direct analogy to shallow-water and hydraulic flows, but Coulomb friction was introduced to model the resistance to motion as the grains slide over a rough slope. The basic shallow-water structure of the governing equations persists today although the theory has been extended to incorporate a simplified Mohr-Coulomb internal rheology (Savage \& Hutter 1989), pore-pressure mobility (Iverson 1997; Denlinger \& Iverson 2001), complex frictional effects at shallow angles (Pouliquen 1999) and topographic features and obstacles (Gray, Wieland \& Hutter 1999b; Wieland, Gray \& Hutter 1999; Gray, Tai \& Noelle 2003).

The study of flow past obstacles is particularly important because shock waves, stationary dead zones and grain-free (vacuum) regions are generated, which can be used to protect both people and infrastructure from natural hazards. Gray et al. $(1999 a ; 2003)$ used small-scale experiments to show how a pyramidal obstacle can generate two oblique shocks, with a sharp increase in the downstream thickness and a decrease in magnitude and rotation of the velocity, so that the flow is parallel to the faces of the pyramid. On the lee-side the flow expanded to form a grain-free vacuum region, which Gray et al. (1999a) used to protect a scale model of the Schneeferner Haus on the Zugspitze. While this prototype defence was never built, there is now strong evidence from field observations (Jóhannesson 2001; Cui, Gray \& Jóhanneson 2007) that oblique shocks were formed as two snow avalanches were deflected by a dam, built to protect the small town of Flateyri in Northwestern Iceland. Even though these flows were in the run-out zone and were being decelerated, they both hit the dam with a Froude number close to 10 and, downstream of the shock, produced a thicker stream that flowed parallel to the dam and eventually led to enhanced run-out. The dams at Flateyri were designed using a very simple point-mass model for the loss of kinetic energy as a single particle runs up the dam wall (Sigurdsson, Tomasson \& Sandersen 1998), which is not very representative of the underlying physics. It is therefore of considerable practical interest to understand how oblique shocks develop in dense granular free-surface flows, what effects can be generated and how well these agree with classical oblique-shock theory. In particular, it is important to understand how the presence of source terms due to gravitational and frictional forcing modify the flow height along the length of the dam, so that the design of defensive dams can be improved. 


\subsection{Hydraulic-type models for granular avalanches}

Most theories modelling granular free-surface flows exploit the shallowness of the avalanche to derive a simplified depth-integrated system of equations for the avalanche thickness $\tilde{h}$ and the depth-averaged velocity components $\tilde{u}, \tilde{v}$ in the downslope and cross-slope directions $\tilde{x}, \tilde{y}$, respectively. In this paper we use a relatively simple theory that assumes the flow is incompressible, with an isotropic lithostatic-pressure distribution through its depth and Coulomb sliding friction at the base (e.g. Grigorian et al. 1967; Kulikovskii \& Eglit 1973; Gray et al. 2003). It is convenient to solve these equations by introducing non-dimensional variables (without tildes) with the following scalings:

$$
\tilde{h}=H h, \quad(\tilde{x}, \tilde{y})=L(x, y), \quad(\tilde{u}, \tilde{v})=\sqrt{L g}(u, v), \quad \tilde{t}=\sqrt{(L / g)} t,
$$

where $H$ is a typical avalanche thickness, $L$ a typical length and $g$ is the constant of gravitational acceleration. The non-dimensional mass and momentum conservation laws become

$$
\begin{aligned}
\frac{\partial h}{\partial t}+\frac{\partial}{\partial x}(h u)+\frac{\partial}{\partial y}(h v) & =0, \\
\frac{\partial}{\partial t}(h u)+\frac{\partial}{\partial x}\left(h u^{2}\right)+\frac{\partial}{\partial y}(h u v)+\frac{\partial}{\partial x}\left(\frac{1}{2} \varepsilon h^{2} \cos \zeta\right) & =h S_{(x)}, \\
\frac{\partial}{\partial t}(h v)+\frac{\partial}{\partial x}(h u v)+\frac{\partial}{\partial y}\left(h v^{2}\right)+\frac{\partial}{\partial x}\left(\frac{1}{2} \varepsilon h^{2} \cos \zeta\right) & =h S_{(y)},
\end{aligned}
$$

where the aspect ratio $\varepsilon=H / L$ and $\zeta$ is the slope inclination angle. The source terms $S_{(x)}$ and $S_{(y)}$ on the right-hand side are composed of the downslope component of gravity, the Coulomb basal friction, which opposes the direction of motion, and the topography gradients:

$$
\begin{aligned}
& S_{(x)}=\sin \zeta-\mu(u /|\boldsymbol{u}|) \cos \zeta-\varepsilon \frac{\partial b}{\partial x} \cos \zeta, \\
& S_{(y)}=-\mu(v /|\boldsymbol{u}|) \cos \zeta-\varepsilon \frac{\partial b}{\partial y} \cos \zeta,
\end{aligned}
$$

where $\mu$ is the coefficient of friction and $b$ is the height of the topography normal to the chute. The equations are non-strictly hyperbolic with characteristic wave speed $c=\sqrt{\varepsilon h \cos \zeta}$ and are very closely related to the Euler equations of gasdynamics and the St Venant or shallow-water equations of hydrodynamics. The ratio of the flow speed and the wave speed defines the Froude number

$$
F r=|\boldsymbol{u}| / \sqrt{\varepsilon h \cos \zeta} .
$$

Flows are supercritical if $F r>1$, critical if $F r=1$ and subcritical if $F r<1$. The hyperbolic structure implies that, in supercritical flows, shock waves can develop with discontinuities in the flow thickness and velocity along a singular surface. In this case the smoothness requirements that are normally used to derive the field equations from the integral conservation laws are no longer satisfied. Instead a limiting procedure (see e.g. Chadwick 1976) must be used to derive the mass and momentum jump conditions

$$
\begin{aligned}
\llbracket h\left(\boldsymbol{u} \cdot \boldsymbol{n}-v_{n}\right) \rrbracket & =0, \\
\llbracket h \boldsymbol{u}\left(\boldsymbol{u} \cdot \boldsymbol{n}-v_{n}\right) \rrbracket+\llbracket \frac{1}{2} \varepsilon h^{2} \cos \zeta \rrbracket \boldsymbol{n} & =0,
\end{aligned}
$$


across the singular surface; $\boldsymbol{n}$ is the normal, $v_{n}$ is the normal propagation speed and the jump bracket is the difference in value of the enclosed variable on either side, i.e. $\llbracket f \rrbracket=f_{1}-f_{2}$, where the subscripts 1 and 2 denote before and after the shock. Note that the jump conditions at the singular surface are independent of the source terms.

\subsection{Granular shock waves in dense chute flows}

Savage (1979) and Brennen, Sieck \& Paslaski (1983) were the first to observe stationary granular shocks in dense chute flows that were analogous to hydraulic jumps in shallow-water theory (e.g. Stoker 1957). More recently, Gray \& Hutter (1997, 1998), Gray \& Tai (1998) and Gray et al. (2003) have observed propagating normal shocks in stratification experiments that are the exact granular equivalent of hydrodynamic bores. These shock waves form with a wide variety of granular materials, including polystyrene beads (Savage 1979), mustard seeds (Brennen et al. 1983), non-pareille sugar grains (hundreds and thousands or Sprinkles) and plastic pellets (Gray et al. 2003), sand, glass balotini (Brennen et al. 1983; Hákonardóttir \& Hogg 2005) and mixtures of different-sized and different-density particles, such as sugar with spherical iron powder (Gray \& Hutter 1997) and sugar and poppy seeds (Gray \& Hutter 1998). The macroscopic properties of the shock appear to be only weakly affected by the size, shape and density of the particles, because there is sufficient dilatation, in these high-solid-fraction flows, to allow the grains to move easily relative to one another. The shocks can be very sharp, occurring over a few grain diameters at high Froude numbers $(F r>5)$, while they can be quite diffuse for $F r \sim 1-2$.

This paper focuses on oblique granular shocks in dense free-surface flows, which have been observed by Gray et al. (1999a), Irmer et al. (1999), Gray et al. (2003) and Hákonardóttir \& Hogg (2005). Oblique shocks also occur in granular gases (e.g. Rericha et al. 2002; Heil et al. 2004; Amarouchene \& Kellay 2006) and these are, in fact, much closer to the oblique shocks in gasdynamics, where much of the original theory was formulated, since the discontinuities are in the velocity and density rather than in the velocity and flow thickness. Figure 1 illustrates the classical oblique-shock solution from gasdynamics (e.g. Ames Research Staff 1953; Saad 1993; Aksel \& Eralp 1994) for polytropic gas constant $\gamma=2$. It is assumed that there is a steady uniform incoming flow with density $\rho_{1}$ and velocity $\boldsymbol{u}_{1}$, which is deflected by an inclined surface at an angle $\theta$ to the oncoming flow to form a straight oblique shock at an angle $\beta$ and a steady uniform outgoing flow with density $\rho_{2}$ and velocity $\boldsymbol{u}_{2}$ parallel to the deflector, as shown in the inset diagram. There are essentially three possibilities. For sufficiently large inflow Mach numbers $M a_{1}$ and small deflection angles $\theta$ there are two solutions for a shock angle $\beta$. The smaller value is called a weak shock, while the larger one is termed a strong shock. These are shown using solid lines and shortdashed lines in figure 1 for various values of $M a_{1}$. If, however, the Mach number decreases or the deflection angle becomes too large then there are no solutions and the shock detaches from the tip of the inclined surface to form a detached oblique shock. The long-dashed line in figure 1 marks the transition between weak and strong shocks and determines the maximum deflection angle $\theta_{d}$, while the dot-dashed line is the sonic line, where the outflow Mach number $M a_{2}=1$. Strong shocks therefore always involve a transition from supersonic to subsonic flow, while weak shocks may be sub- or supersonic after the shock. This is of great importance, as it determines the appropriate number of boundary conditions that must be imposed in both physical experiments and numerical simulations in order to realize the shocks. Strong shocks require significantly increased downstream pressure to form and as a result tend to be 


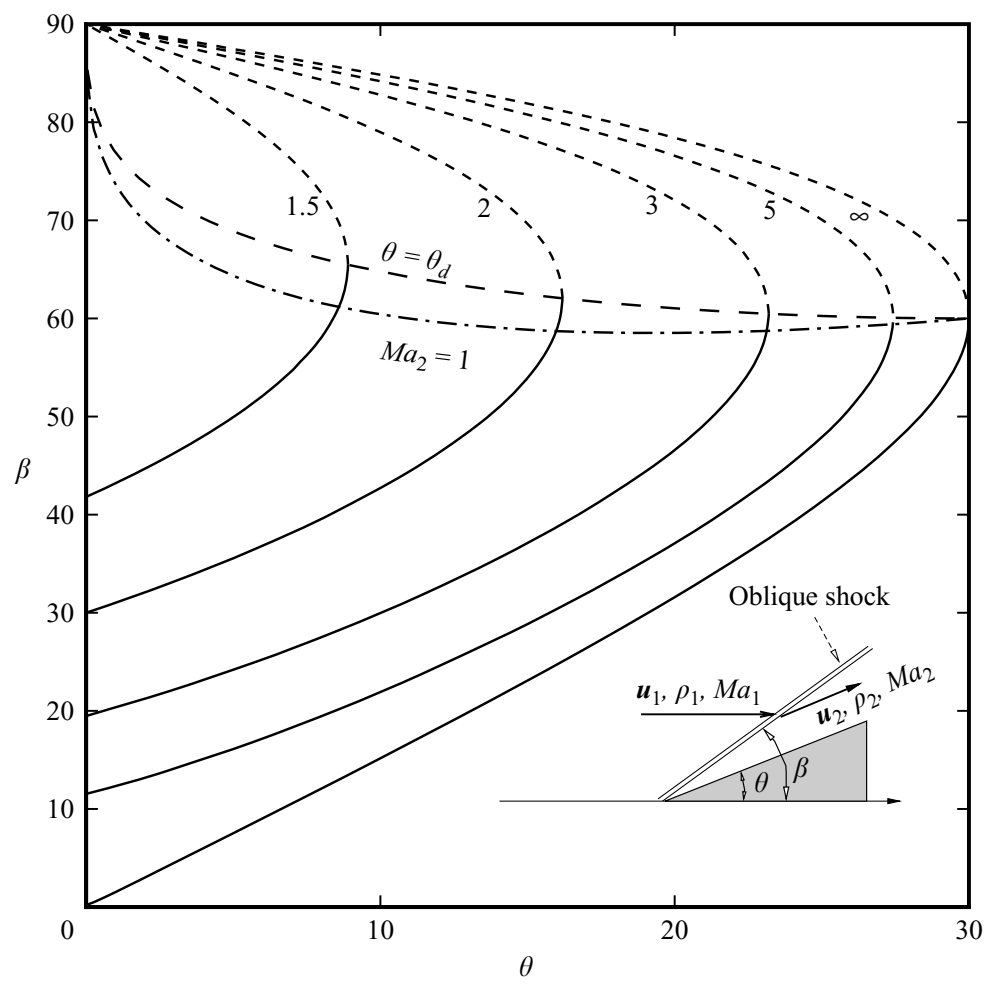

FIGURE 1. Shock angle $\beta$ as a function of the flow-deflection angle $\theta$ for inflow Mach numbers $M a_{1}=1.5,2,3,5, \infty$. The flow is assumed to be isentropic with polytropic gas constant $\gamma=2$. Weak shocks are denoted by the solid lines and the strong shocks by the short-dashed lines. The maximum-deflection-angle line $\theta_{d}$ (long-dashed) marks the transition between the weak and strong shocks; it reaches a maximum of $30^{\circ}$. The sonic line (dot-dashed) corresponds to an outflow Mach number $M a_{2}=1$. The inset gives a schematic diagram of the oblique shock, showing the shock and deflection angles and the states on either side of the shock.

observed in internal flows, within engines and nozzles. Weak shocks, however, tend to form naturally in external flows.

In this paper we show for the first time how strong shocks can be triggered in dense granular chute flows by careful control of the downstream conditions. This produces a flow with a very large increase in the downstream flow thickness and a corresponding decrease in velocity, which dissipates much more energy than a weak shock. In addition, we show how a detached shock can form experimentally, if the wedge angle is sufficiently large or the Froude number is sufficiently low, and demonstrate that the detachment angle is in excellent agreement with the values predicted by classical oblique-shock theory. A shock-capturing numerical method is then used to solve the full system (1.2)-(1.6), in order to quantify the effect that the source terms have on the shock angle and the downstream flow thickness for both accelerative and decelerative slopes. This has important implications for the design of defensive dams used to divert hazardous geophysical mass flows.

\section{Oblique hydraulic jumps in the absence of source terms}

The theory of oblique hydraulic jumps differs from that of gasdynamics and is much less well known. In this section the original solution developed by Rouse 
(1938), Ippen (1949) and Rouse (1949) is reviewed, and new explicit results for the maximum-deflection-angle curve and the critical line are derived. The classical solution is only valid when the source terms (1.5) and (1.6) are identically zero in the field equations, which is only ever approximately satisfied for granular flows. It is nevertheless a useful approximation for high-Froude-number flows where the slope is almost non-accelerative and $u \gg v$. The classical solution is derived from the mass-jump condition (1.8) and the tangential and normal components of the momentum-jump condition (1.9), which are

$$
\begin{aligned}
h_{1}\left|\boldsymbol{u}_{1}\right| \sin \beta & =h_{2}\left|\boldsymbol{u}_{2}\right| \sin (\beta-\theta), \\
h_{1} \boldsymbol{u}_{1}^{2} \sin \beta \cos \beta & =h_{2} \boldsymbol{u}_{2}^{2} \sin (\beta-\theta) \cos (\beta-\theta), \\
\frac{1}{2} \varepsilon h_{1}^{2} \cos \zeta+h_{1} \boldsymbol{u}_{1}^{2} \sin ^{2} \beta & =\frac{1}{2} \varepsilon h_{2}^{2} \cos \zeta+h_{2} \boldsymbol{u}_{2}^{2} \sin ^{2}(\beta-\theta) .
\end{aligned}
$$

By substituting for $\left|\boldsymbol{u}_{2}\right| \sin (\beta-\theta)$ from (2.1) into (2.3) and solving for $\sin \beta$ we obtain

$$
\sin \beta=\frac{1}{F r_{1}} \sqrt{\frac{1}{2} \frac{h_{2}}{h_{1}}\left(1+\frac{h_{2}}{h_{1}}\right),}
$$

which Gray et al. (2003) showed to be in very good agreement with measured weakshock angles, even on slopes with moderate acceleration. Squaring both sides of (2.4) and solving the resultant quadratic equation for $h_{2} / h_{1}$ implies that

$$
\frac{h_{2}}{h_{1}}=\frac{1}{2}\left(\sqrt{1+8 F r_{1}^{2} \sin ^{2} \beta}-1\right) .
$$

An alternative expression for the thickness ratio can be obtained by eliminating $\left|\boldsymbol{u}_{2}\right|$ between (2.1) and (2.2) to show that

$$
\frac{h_{2}}{h_{1}}=\frac{\tan \beta}{\tan (\beta-\theta)} .
$$

Using the trigonometric identity $\tan (\beta-\theta)=(\tan \beta-\tan \theta) /(1+\tan \beta \tan \theta)$, solving (2.6) for $\tan \theta$ and substituting for the thickness ratio from (2.5) gives an expression for the wedge angle $\theta$ as a function of the inflow Froude number $F r_{1}$ and the shock angle $\beta$ :

$$
\tan \theta=\frac{\tan \beta\left(\sqrt{1+8 F r_{1}^{2} \sin ^{2} \beta}-3\right)}{2 \tan ^{2} \beta-1+\sqrt{1+8 F r_{1}^{2} \sin ^{2} \beta}} .
$$

This is the classical form of the solution given by Ippen (1949) and is plotted in figure 2 . Qualitatively it is very similar to the textbook shock-angle curve from gasdynamics illustrated in figure 1. Again there are three possibilities. Provided that the inflow Froude number $F r_{1}$ is large enough, there are two solutions for the shock angle $\beta$ for any given deflection angle $\theta$. In the terminology of gasdynamics the lower value is called a weak shock and the larger angle a strong shock. If the Froude number is too low, there are no solutions and the shock detaches from the front of the wedge. There are, however, some marked differences from gasdynamics. As $F r_{1} \longrightarrow \infty$ the weak-shock angle $\beta \longrightarrow \theta$ and the strong shock tends to a normal shock, $\beta \longrightarrow \pi / 2$. All solutions are therefore contained in the top triangular region of figure 2 . In contrast, figure 1 shows that the strong shock only approaches a normal shock for $\theta=0$ and the maximum deflection angle never exceeds $30^{\circ}$. This is emphasized in the inset diagram, which shows the $F r_{1} \longrightarrow \infty$ curve and the $M a_{1} \longrightarrow \infty$ curve on 


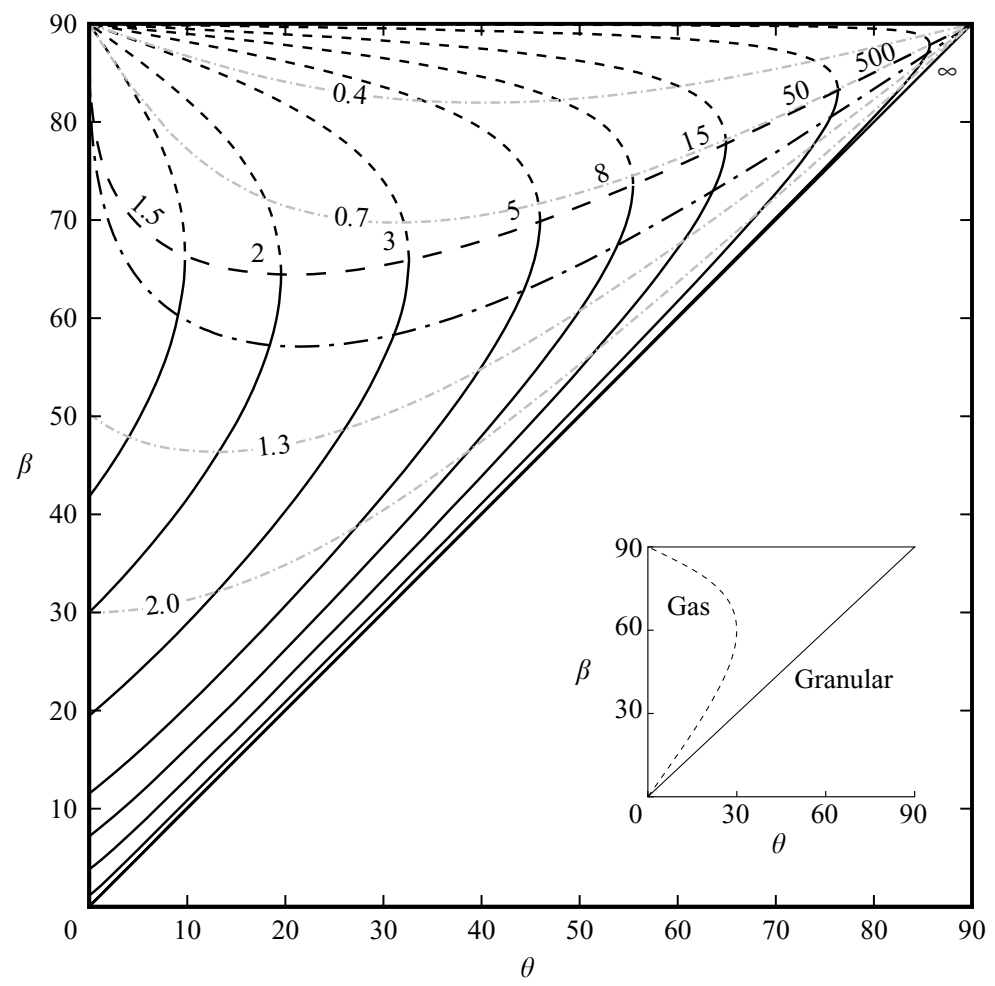

FIGURE 2. The shock angle $\beta$ is plotted as a function of the wedge or deflection angle $\theta$ for $F r_{1}=1.5,2,3,5,8,15,50,500, \infty$. Weak shocks are denoted by the solid lines and strong shocks by the short-dashed lines. The maximum deflection angle $\theta_{d}$ marks the transition between the weak and strong shocks and is denoted by the long-dashed line. The dot-dashed line denotes the critical line, where $F r_{2}=1$. The light-grey dot-dashed lines show the values of $\mathrm{Fr}_{2}$ after the shock. Inset is a plot showing the limiting curves for the granular (solid) and gasdynamic (dashed) cases as $F r_{1} \longrightarrow \infty$ and $M a_{1} \longrightarrow \infty$, respectively.

the same scale for comparison. Note that when $\theta=0$ the weak-shock angle decreases to $\beta_{\text {min }}=\sin ^{-1}(1 / F r)$, which is the granular equivalent of a Mach wave, while the strong shock strengthens to a normal shock with $\beta=\pi / 2$ as in gasdynamics.

Explicit formulae exist in gasdynamics for both $\theta_{d}$ and the critical curve (e.g. Ames Research Staff 1953; Saad 1993; Aksel \& Eralp 1994) and it is of interest to derive similar formulae for granular flows. A slightly different form of the shock-angle relation (2.7), which is more amenable to further manipulation, is obtained using (2.1) and (2.6) to substitute for $h_{2}$ and $\left|\boldsymbol{u}_{2}\right|$ in (2.3). Gathering the terms multiplied by $F r_{1}$ together and dividing through by $\tan \beta-\tan (\beta-\theta)$ yields a quadratic equation for $\tan (\beta-\theta)$. An alternative formula for the wedge or deflection angle is therefore given by the relation

$$
\theta=\beta-\tan ^{-1}\left(\frac{1+\sqrt{1+8 F r_{1}^{2} \sin ^{2} \beta}}{2 F r_{1}^{2} \sin (2 \beta)}\right) .
$$

An explicit solution for the detachment angle $\theta_{d}$ can be found by differentiating (2.8) with respect to $\beta$, equating the result to zero and making the substitution $\xi=\sin ^{2} \beta_{d}$. 


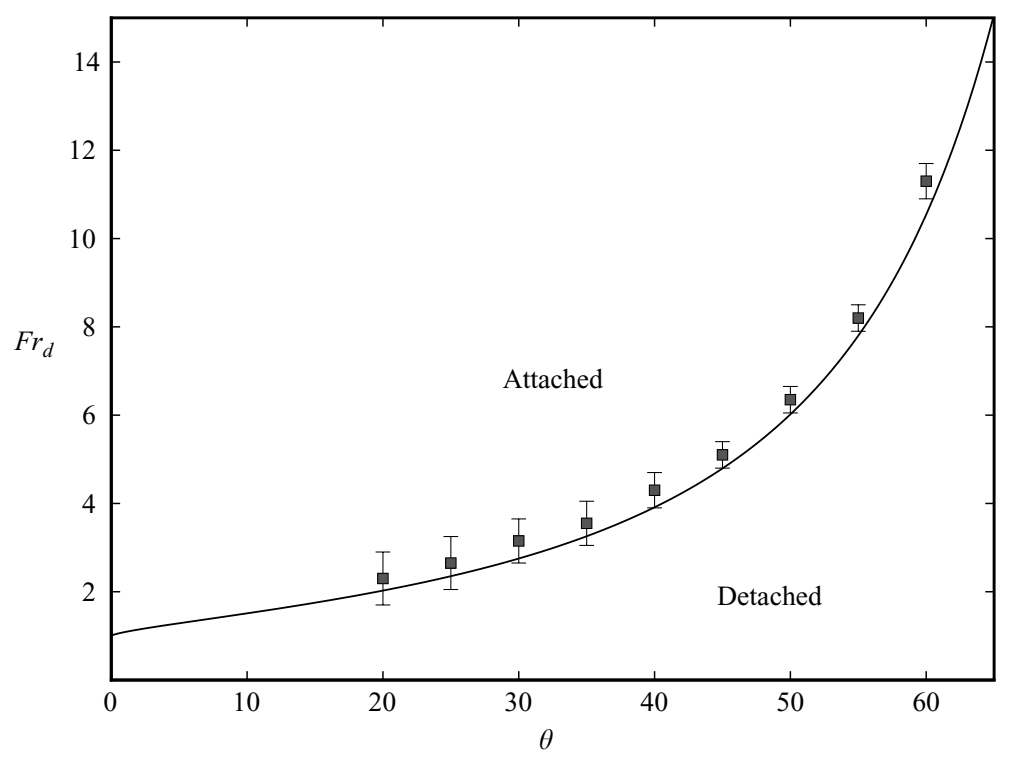

FIGURE 3. The theoretical detachment Froude number $F r_{d}$ as a function of the deflection angle $\theta$ (solid line). For values of $F r_{1}>F r_{d}$ the oblique shock is attached, but it detaches when $F r_{1}<F r_{d}$. The solid squares and the error bars indicate the experimentally measured detachment Froude number on a slope inclined at $\zeta=38^{\circ}$ to the horizontal.

After some manipulation this yields a quartic equation for $\xi$ :

$16 F r_{1}^{6} \xi^{4}-\left(32 F r_{1}^{6}+6 F r_{1}^{4}\right) \xi^{3}+\left(16 F r_{1}^{6}-12 F r_{1}^{4}\right) \xi^{2}+\left(5 F r_{1}^{2}+10 F r_{1}^{4}\right) \xi+1+2 F r_{1}^{2}=0$.

This can be solved exactly using the formula of Ferrari and Cardano for quartic equations (e.g. Abramowitz \& Stegun 1970), which for brevity we do not repeat here. For practical purposes it is easier to use one of the many numerical root-finding techniques to solve (2.9). The maximum deflection angle is therefore calculated by first constructing $\beta_{d}=\sin ^{-1}\left(\xi^{1 / 2}\right)$ and substituting the result into either of the deflectionangle relations (2.7) and (2.8). The solution $\left(\theta_{d}, \beta_{d}\right)$ is parameterized by the detachment Froude number $F r_{d}$ and marks the transition between strong and weak shocks. It is shown on figure 2 as the long-dashed line and connects the points where $\mathrm{d} \theta / \mathrm{d} \beta=0$. Figure 3 shows the detachment Froude number $F r_{d}$ plotted as a function of the wedge or deflection angle $\theta$ and, for comparison, the experimentally measured angles. The theoretical and experimental results are in close agreement. The curve is therefore very useful as, for a given wedge or deflection angle $\theta$, it accurately predicts that the shock stays attached for $F r_{1}>F r_{d}$ and that it detaches when $F r_{1}<F r_{d}$.

An expression for the ratio of the velocity magnitudes before and after the shock is given by eliminating $h_{2}$ between (2.1) and (2.6) to give

$$
\frac{\left|\boldsymbol{u}_{2}\right|}{\left|\boldsymbol{u}_{1}\right|}=\frac{\cos \beta}{\cos (\beta-\theta)} \text {. }
$$

It follows that the ratio of the Froude numbers satisfies

$$
\frac{F r_{2}^{2}}{F r_{1}^{2}}=\frac{\cos ^{3} \beta \sin (\beta-\theta)}{\sin \beta \cos ^{3}(\beta-\theta)}=\frac{\tan (\beta-\theta)\left(1+\tan ^{2}(\beta-\theta)\right)}{\tan (\beta)\left(1+\tan ^{2}(\beta)\right)} .
$$




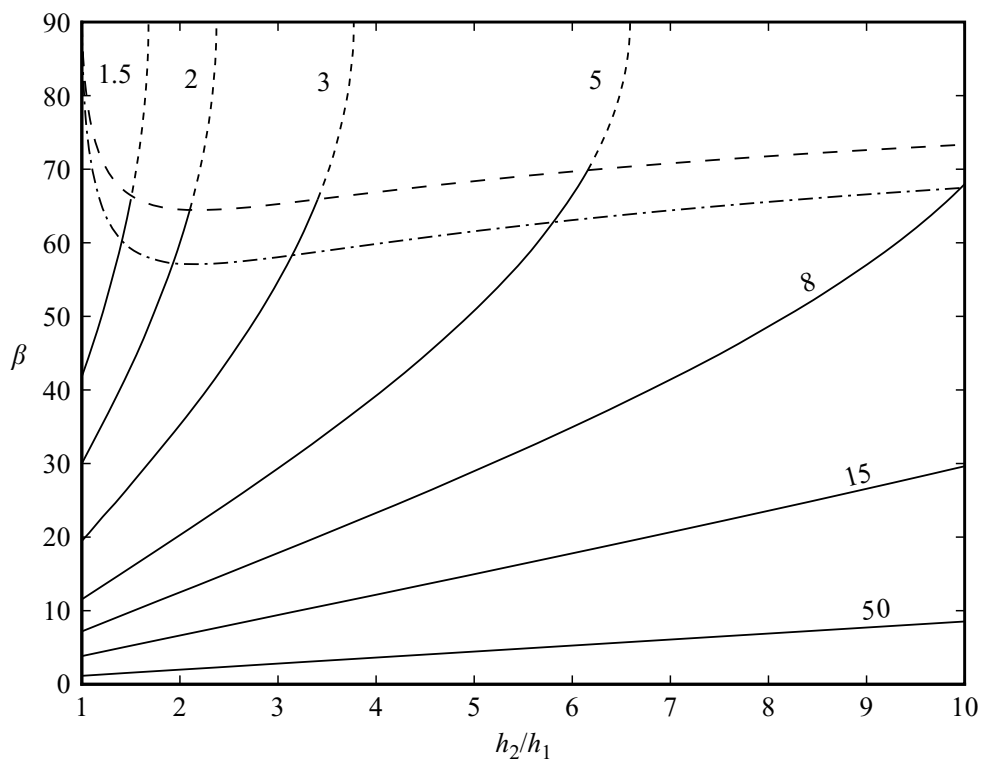

FIGURE 4. The shock angle $\beta$ is plotted as a function of the thickness ratio $h_{2} / h_{1}$ for $F r_{1}=1.5,2,3,5,8,15,50$. Weak shocks are denoted by the solid lines and strong shocks by the short-dashed lines. The maximum-deflection-angle line, which marks the transition between the weak and strong shocks, is long-dashed. The dot-dashed line corresponds to $\mathrm{Fr}_{2}=1$.

An explicit solution for the critical line, where $F r_{2}=1$, is found by substituting for $\tan (\beta-\theta)$ from $(2.8)$ and then using trigonometric identities to substitute $\xi=\sin ^{2} \beta_{c}$. After some manipulation this yields a cubic equation for $\xi$ :

$$
4 F r_{1}^{6} \xi^{3}-\left(8 F r_{1}^{6}+16 F r_{1}^{4}\right) \xi^{2}+\left(6 F r_{1}^{2}+4 F r_{1}^{6}+8 F r_{1}^{4}\right) \xi+F r_{1}^{2}+1=0
$$

which can be solved explicitly using Cardano's formula (e.g. Abramowitz \& Stegun 1970). The critical curve is given by calculating $\beta_{c}=\sin ^{-1}\left(\xi^{1 / 2}\right)$ and substituting into either (2.7) or (2.8) to give $\theta_{c}$. The curve $\left(\theta_{c}, \beta_{c}\right)$ is also parameterized by the Froude number and is shown in figure 2 as a dot-dashed line. As for the maximum deflection angle, the endpoints lie at $(\pi / 2,0)$ and $(\pi / 2, \pi / 2)$ but $\beta_{c}<\beta_{d}$ for $\theta \in(0, \pi / 2)$. It follows that strong shocks always involve a transition from super- to subcritical flow, just as in gasdynamics, while weak shocks can be either sub- or supercritical after the shock.

The same procedure can be used to construct explicit solutions for other values of $F r_{2}$. The general case yields a cubic equation for $\xi=\sin ^{2} \beta$, i.e.

$$
c_{3} \xi^{3}-c_{2} \xi^{2}+c_{1} \xi+c_{0}=0
$$

where the coefficients are

$$
\left.\begin{array}{rrr}
c_{3} & =4 F r_{1}^{6}, & c_{2}=4 F r_{2}^{2} F r_{1}^{4}+8 F r_{2}^{4} F r_{1}^{4}+4 F r_{1}^{4}+8 F r_{1}^{6}, \\
c_{1}=6 F r_{1}^{2} F r_{2}^{2}+4 F r_{2}^{2} F r_{1}^{4}+4 F r_{1}^{4}+4 F r_{1}^{6}, & c_{0}=F r_{2}^{2}+F r_{1}^{2} .
\end{array}\right\}
$$

The corresponding solutions are illustrated as light-grey dot-dashed lines on figure 2. The value of the thickness ratio can be calculated from (2.6) and that of the velocity-magnitude ratio from (2.10). These are illustrated graphically in figures 4 and 5. Given the Froude number and the deflection angle, two values of $\beta$ can be determined from figure 2 and then used in figure 4 to determine the corresponding 


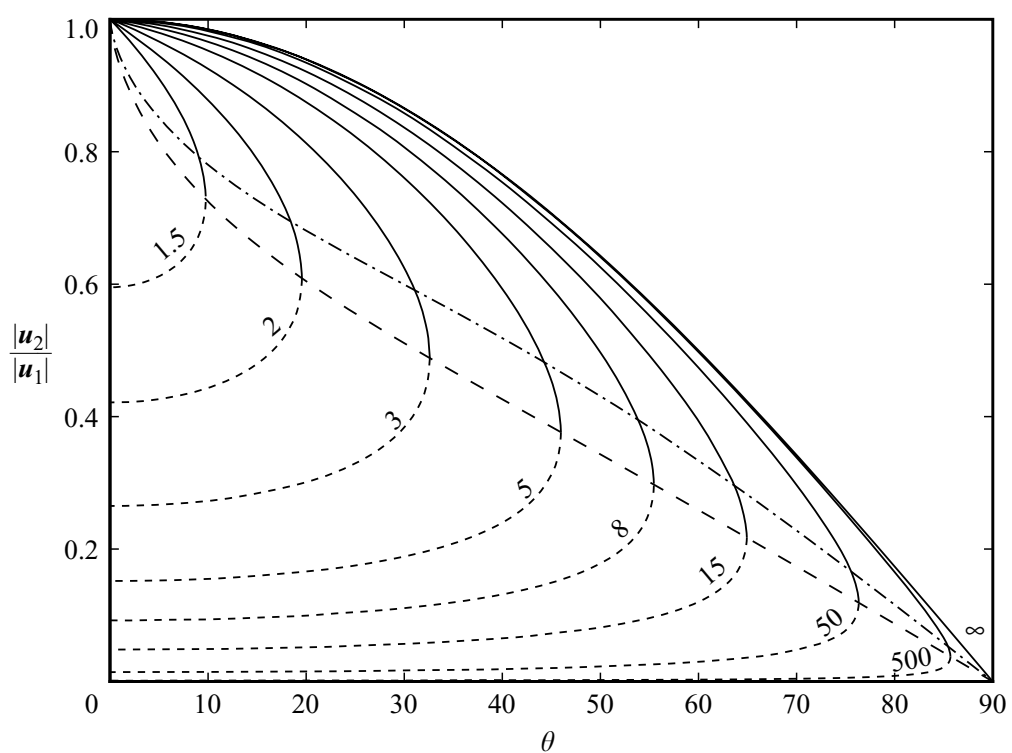

FIGURE 5. The ratio of the velocity magnitudes $\left|\boldsymbol{u}_{2}\right| /\left|\boldsymbol{u}_{1}\right|$ is plotted as a function of the wedge angle $\theta$ for $F r_{1}=1.5,2,3,5,8,15,50,500, \infty$. Weak shocks are denoted by solid lines and strong shocks by short-dashed lines. The maximum-deflection-angle line, which marks the transition between weak and strong shocks, is long-dashed. The dot-dashed line corresponds to $F r_{2}=1$.

thickness jump. The thickness jump is significantly larger for strong shocks than for weak shocks, the magnitude of the jump increasing with increasing $F r_{1}$. The height of a retaining dam or wall must therefore be significantly higher if a strong shock is generated, to prevent the flow from overtopping. Figure 5 shows that the velocity jump is also less for weak shocks than for strong shocks, the magnitude of the jump becoming stronger as the deflection angle increases.

\section{Experimental weak, strong and detached oblique granular shocks}

Weak oblique shocks have been observed experimentally in dense granular freesurface flows by Gray et al. (1999a), Gray et al. (2003) and Hákonardóttir \& Hogg (2005), and the measured shock angles are in good agreement with the weakshock angles predicted by (2.4) and (2.7) (Irmer et al. 1999; Gray et al. 2003; Hákonardóttir \& Hogg 2005). However, it has not previously been clear how to generate strong shocks in dense granular free-surface flows. We will describe a simple procedure by which they can be triggered. We will also show that the simple solution for the detachment angle $\theta_{d}$ in (2.9) is in excellent agreement with experimental values over a wide range of Froude numbers.

All the experiments were performed on a chute $300 \mathrm{~mm}$ wide and $450 \mathrm{~mm}$ long, inclined at $38^{\circ}$ to the horizontal. A large hopper was situated at the top of the chute, whose exit was controlled by a double gate: the first gate is fixed and is preset to a given outflow height to control the mass flux, while the second allows easy release. Once this second gate is raised the particles flow rapidly down the chute, quickly establishing a steady non-uniform flow in which the avalanche accelerates and the thickness decreases slowly with downstream distance. The flow velocity is measured using particle image velocimetry (PIV) equipment and the thickness is measured 


\begin{tabular}{ccc} 
& \multicolumn{2}{c}{ Weak shock } \\
\cline { 2 - 3 } & Experiment & Theory \\
$\tilde{h}_{2}$ & $27 \pm 1 \mathrm{~mm}$ & $28.2 \mathrm{~mm}$ \\
$\left|\tilde{\boldsymbol{u}}_{2}\right|$ & $1.13 \pm 0.01 \mathrm{~m} \mathrm{~s}^{-1}$ & $1.15 \mathrm{~m} \mathrm{~s}^{-1}$ \\
$h_{2}$ & $0.9 \pm 0.03$ & 0.94 \\
$\left|\boldsymbol{u}_{2}\right|$ & $2.1 \pm 0.02$ & 2.13 \\
$F r_{2}$ & $2.24 \pm 0.06$ & 2.23 \\
$\beta$ & $29^{\circ} \pm 1^{\circ}$ & $30.69^{\circ}$
\end{tabular}

\begin{tabular}{cc}
\multicolumn{2}{c}{ Strong shock } \\
\hline Experiment & Theory \\
$60 \pm 1.8 \mathrm{~mm}$ & $59.1 \mathrm{~mm}$ \\
$0.18 \pm 0.01 \mathrm{~m} \mathrm{~s}^{-1}$ & $0.21 \mathrm{~m} \mathrm{~s}^{-1}$ \\
$2.0 \pm 0.06$ & 1.97 \\
$0.35 \pm 0.02$ & 0.4 \\
$0.24 \pm 0.02$ & 0.28 \\
$78^{\circ} \pm 1^{\circ}$ & $86.15^{\circ}$
\end{tabular}

TABLE 1. Comparison of the experimental and predicted flow thicknesses, velocities, Froude numbers and shock angles for the weak and strong oblique shocks illustrated in figure 6 . The predicted values are for a wedge angle $\theta=20^{\circ}$, chute angle $\zeta=38^{\circ}$, inflow velocity magnitude $\left|\tilde{\boldsymbol{u}}_{1}\right|=1.32 \mathrm{~m} \mathrm{~s}^{-1}$ and thickness $\tilde{h}_{1}=9 \mathrm{~mm}$, giving $F r_{1}=5$. These values are equivalent to a non-dimensional thickness $h_{1}=0.30$ and velocity $\left|\boldsymbol{u}_{1}\right|=2.43$ with scalings $H=L=3 \mathrm{~cm}$. The experimental and predicted non-dimensional thicknesses and velocities are shown in the middle two rows.

using a scale along the transparent sidewall. The flow is deflected by a wedge of length $240 \mathrm{~mm}$ placed $225 \mathrm{~mm}$ downstream, which can be inclined at any angle $\theta$. The avalanche itself is composed of roughly spherical $1 \mathrm{~mm}$ diameter red-and-white non-pareille sugar grains, commonly known as 'hundreds and thousands' or Sprinkles, which can be obtained from Werner's Dragéefabrik, Tornesch, Germany. These grains produce very sharp, clearly defined, shocks even at moderate Froude numbers.

\subsection{Weak shocks}

An example of a weak shock is shown in the perspective and overhead photographs in the top panels of figure 6. At the tip of the wedge the avalanche is $9 \mathrm{~mm}$ thick and moves at approximately $1.32 \mathrm{~m} \mathrm{~s}^{-1}$. Using the scalings (1.1) with equal vertical and horizontal length scales $H=L=3 \mathrm{~cm}$, non-dimensional thickness $h_{1}$ is 0.3 and the velocity magnitude $\left|\boldsymbol{u}_{1}\right|$ is 2.43 , which implies that the incoming Froude number $F r_{1}$ equals 5, by (1.7). A weak oblique shock forms naturally in this configuration and lies at an angle of approximately $29^{\circ}$ to the downstream direction. The streak lines show that the flow is approximately parallel to the wedge after the shock. The avalanche thickness jumps to approximately $27 \mathrm{~mm}$, while the velocity magnitude decreases to $1.13 \mathrm{~m} \mathrm{~s}^{-1}$. In non-dimensional units, $h_{2}=0.9$ and $\left|\boldsymbol{u}_{2}\right|=2.1$. Using a wedge angle $\theta=20^{\circ}$ and incoming Froude number $F r_{1}=5$, (2.6), (2.7) and (2.10) predict that the downstream thickness $h_{2}=0.94$, the velocity magnitude $\left|\boldsymbol{u}_{2}\right|=2.13$ and the shock angle $\beta=30.69^{\circ}$, i.e. $\tilde{h}_{2}=28.2 \mathrm{~mm}$ and $\left|\tilde{\boldsymbol{u}}_{2}\right|=1.15 \mathrm{~m} \mathrm{~s}^{-1}$. The flow variables are summarized in table 1 . The measured thickness, velocity magnitude and shock angle are within $6 \%$ of those predicted by the theory. This is exceptionally good agreement considering that source terms have been neglected and that an accuracy of $10 \%$ is often the best that can be expected for fluid flows.

\subsection{Strong shocks}

Strong shocks have not been observed before in granular avalanches, and even in hydraulics they seem to be a rare event. Just as in gasdynamics, there must be an increased downstream pressure in order for them to be triggered. To achieve this, a second double gate was placed across the chute at the end of the wedge. The height of the fixed gate was varied by trial and error to obtain the correct outflow mass flux, while the second blocking gate was initially shut at the start of the experiment. Once the avalanche is released from the hopper it flows rapidly downslope to create a 

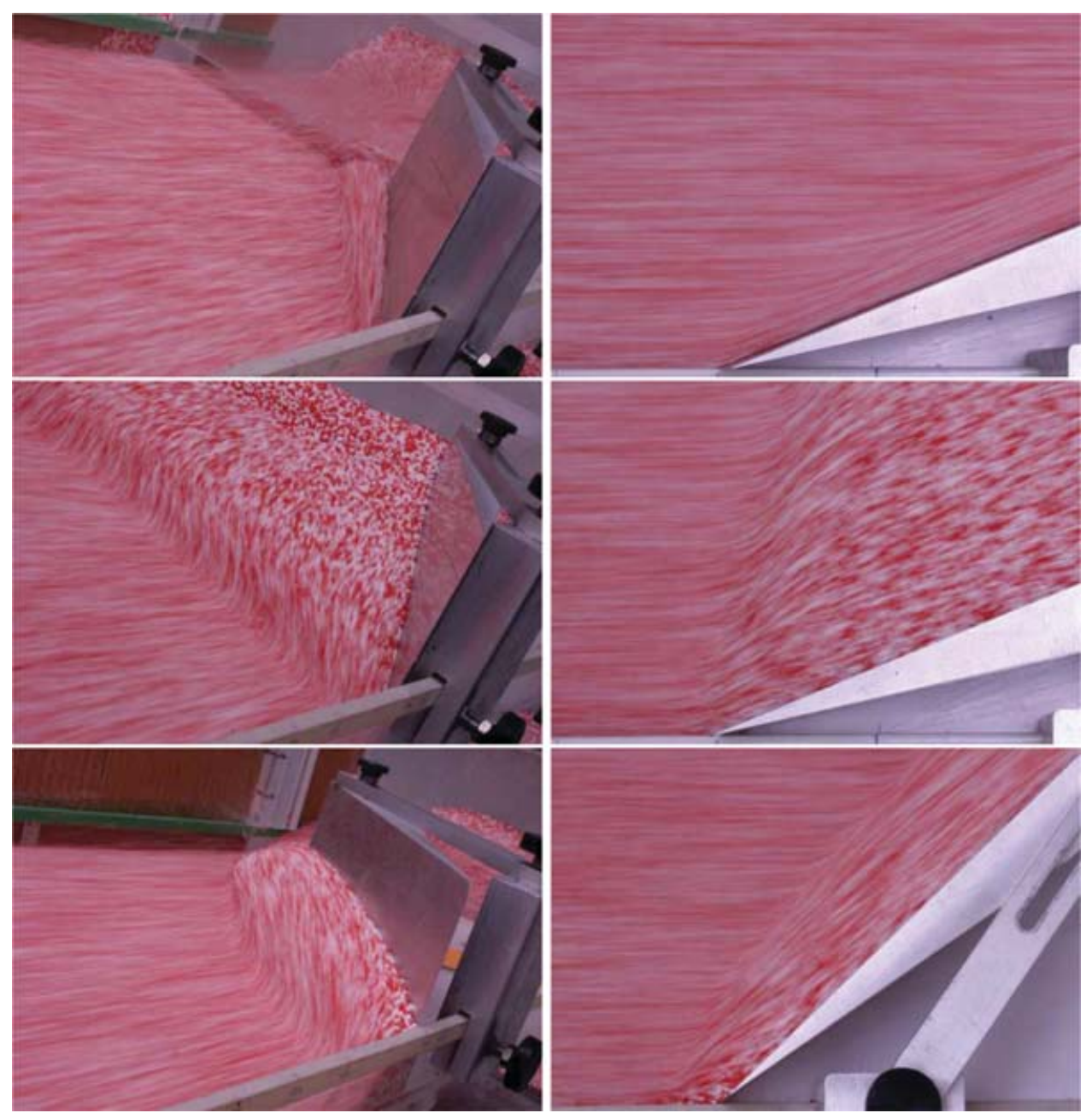

Figure 6. Perspective (on the left) and overhead (on the right) photographs showing that weak (top), strong (middle) and detached (bottom) oblique shocks are formed in dry granular avalanches composed of non-pareille sugar grains (commonly known as 'hundreds and thousands' or Sprinkles). A shutter speed of 1/25 second is used to produce streaks that indicate both the direction of flow and the relative speed of the grains before and after the shock. The downslope direction is from left to right in the overhead photos and the chute is inclined at an angle $\zeta=38^{\circ}$ to the horizontal. The Froude number $F r_{1}$ just before the wedge is equal to 5 and the wedge angle $\theta=20^{\circ}$ for both the weak and strong shocks. The detached shock is generated with $F r_{1}=4$ and $\theta=44^{\circ}$, which lies above the detachment angle $\theta_{d}=40.57^{\circ}$ predicted by the classical theory. The cross-slope width of the overhead panels on the right-hand side is $13.5 \mathrm{~cm}$. Three movies showing the formation of the weak, strong and detached shocks are available with the online version of the paper.

weak oblique shock along the wedge, before hitting the gate and stopping to produce an upslope-travelling strong shock. As the top of the strong shock nears the tip of the wedge the second blocking gate is released and a steady-state strong oblique shock is quickly formed, as shown in the middle panels of figure 6 . A movie showing the formation of the strong shock is available with the online version of the paper. Although the inflow conditions are the same as for the weak shock, the strong shock 
is much steeper, lying at an angle of approximately $78^{\circ}$ right across the chute and intersecting with the far sidewall. On the downstream side of the shock the avalanche increases dramatically in thickness to around $60 \mathrm{~mm}$ and the velocity decreases to $0.18 \mathrm{~m} \mathrm{~s}^{-1}$. These correspond to $h_{2}=2$ and $\left|\boldsymbol{u}_{2}\right|=0.35$ in non-dimensional units. The strong shock for $F r_{1}=5$ and $\theta=20^{\circ}$ is predicted to lie at an angle $\beta=86.15^{\circ}$; the downstream thickness $h_{2}$ is 1.97 and the velocity magnitude $\left|\boldsymbol{u}_{2}\right|$ is 0.4 non-dimensional units, which, correspond to $\tilde{h}_{2}=59.1 \mathrm{~mm}$ and $\left|\tilde{\boldsymbol{u}}_{2}\right|=0.21 \mathrm{~m} \mathrm{~s}^{-1}$. The predicted and measured thicknesses are within $2 \%$ of one another, the velocity magnitudes are within $16 \%$ and the shock angles are $10 \%$ apart. The oblique-hydraulic-shock relations in $\S 2$ therefore still provide useful approximations even in the case of a strong shock. The flow variables and predictions are summarized in table 1. Crucially for dam-height design, the predicted strong-shock thickness is very close to the observed value.

\subsection{Detached oblique shocks}

Detached oblique shocks are much easier to generate and the second set of gates can be discarded. All that is required is either to reduce the incoming Froude number or to increase the wedge angle so that the flow conditions lie to the right of the detachmentFroude-number curve $\mathrm{Fr}_{d}$ in figure 3. A combination of these two changes is achieved by increasing the wedge deflection angle to $\theta=44^{\circ}$ and moving it upstream to a point where the inflow thickness increases to $10.5 \mathrm{~mm}$ and the velocity magnitude decreases to $1.13 \mathrm{~m} \mathrm{~s}^{-1}$, so that $F r_{1}=4$. The corresponding non-dimensional values are $h_{1}=0.35$ and $\left|\boldsymbol{u}_{1}\right|=2.1$. For an inflow Froude number $F r_{1}=4$, (2.8) and (2.9) predict that the shock detaches at $\theta_{d}=40.57^{\circ}$, as shown in figure 3. For a wedge angle $\theta>\theta_{d}$ the shock should be, and is, detached, as shown in the bottom panels of figure 6. An oblique shock starts out as a normal shock located about $19 \mathrm{~mm}(0.63$ non-dimensional units) upstream of the wedge and the particles immediately behind it are almost stationary. The shock angle slowly decreases with downstream distance and bends towards the wedge. By successively moving the position of the wedge on the slope and varying the deflection angle it is possible to determine the detachment angle $\theta_{d}$ experimentally for a range of inflow Froude numbers. The solid squares and error bars in figure 3 show that the experimental detachment angles are in very close agreement with those predicted by the theory. The curve lies slightly below the measured values, which may either be an effect of the source terms or evidence for a non-isotropic pressure distribution (e.g. Savage \& Hutter 1989; Iverson 1997; Denlinger \& Iverson 2001). However, these deviations are relatively small and within, or close to, the experimental error bars over much of the range.

\section{Numerical method}

Gray et al. (2003) developed a numerical scheme to compute the flow past pyramidal obstacles with sloped sides. The presence of the obstacle was included in the source terms (1.5), (1.6) by using gradients of the obstacle height $b$ normal to the inclined reference plane. This approach has the advantage that it allows the flow past complex obstacles to be computed on a simple rectangular grid. However, the method cannot be used for obstacles with walls that are normal to the plane of the chute. The simplest way to compute the flow past the wedge is to introduce a body-fitted coordinate system (e.g. Anderson 1982), such as the one illustrated in figure 7. The advantage of body-fitted coordinates is that they allow the wall boundary conditions to be applied easily and that the grid resolution can be enhanced near the obstacle. 


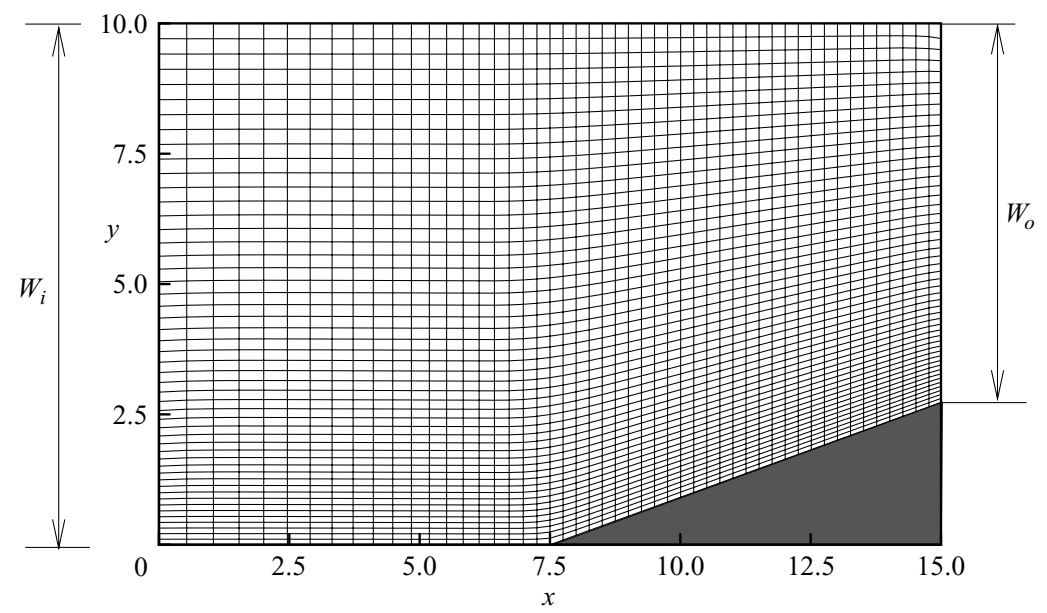

Figure 7. A sketch of the H-grid used in the computation. The bottom right shaded region denotes the wedge. The lateral width of the inflow is $W_{i}$ and of the outflow is $W_{o}$.

\subsection{Body-fitted coordinates}

Introducing conservative variables $h, m=h u$ and $n=h v$, the conservation laws (1.2)-(1.4) are first written in vector form, as

$$
\frac{\partial U}{\partial t}+\frac{\partial E}{\partial x}+\frac{\partial F}{\partial y}=S
$$

where the fluxes and source terms are

$$
\begin{aligned}
U & =(h, m, n)^{T}, \\
E & =\left(m, m^{2} / h+\frac{1}{2} \varepsilon h^{2} \cos \zeta, m n / h\right)^{T}, \\
F & =\left(n, m n / h, n^{2} / h+\frac{1}{2} \varepsilon h^{2} \cos \zeta\right)^{T}, \\
S & =\left(0, h S_{(x)}, h S_{(y)}\right)^{T},
\end{aligned}
$$

and the superscript $T$ denotes the transpose. The chute-coordinate system $O x y$ is mapped to the body-fitted coordinate system $O \xi \eta$ by the transformation

$$
\xi=\xi(x, y), \quad \eta=\eta(x, y), \quad \tau=t,
$$

so that the upper sidewall, as well as the combination of the lower sidewall and wedge, lie along $\eta=$ constant coordinate lines. The mapped system of equations can once again be written in conservative form,

$$
\frac{\partial \hat{U}}{\partial \tau}+\frac{\partial \hat{E}}{\partial \xi}+\frac{\partial \hat{F}}{\partial \eta}=\hat{S}
$$

by defining the fluxes and the source terms as

$$
\hat{U}=J^{-1} U, \quad \hat{E}=J^{-1}\left(\xi_{x} E+\xi_{y} F\right), \quad \hat{F}=J^{-1}\left(\eta_{x} E+\eta_{y} F\right), \quad \hat{S}=J^{-1} S,
$$

where the subscripts denote differentiation with respect to that subscript and the Jacobian $J$ is given by $\xi_{x} \eta_{y}-\xi_{y} \eta_{x}=\left(x_{\xi} y_{\eta}-x_{\eta} y_{\xi}\right)^{-1}$.

\subsection{NT schemes and implementation of the boundary conditions}

The transformed system (4.4) is solved in the computational domain using an explicit two-dimensional non-staggered non-oscillating central scheme developed by Jiang et al. (1998). These high-resolution methods are known as NT schemes, after 


$\begin{array}{lcc}\text { Boundary type } & \text { Number of conditions } & \text { Prescribed variables } \\ \text { supercritical inflow } & 3 & h, u, v \\ \text { supercritical outflow } & 0 & \text { none } \\ \text { subcritical outflow } & 1 & m=h u \\ \text { wall boundary } & 1 & h \boldsymbol{u} \cdot \boldsymbol{n}=0\end{array}$

TABLE 2. A summary of the applied boundary conditions.

Nessyahu \& Tadmor (1990), who first devised them. They have the advantage that they provide a simple Riemann-solver-free recipe that has superior resolution to upwinded methods. Gray et al. (2003) used a two-dimensional staggered NT scheme (Jiang \& Tadmor 1998; Lie \& Noelle 2003) to compute the granular free-surface flow past a pyramidal obstacle. While this method produces excellent results, it is not as convenient for flows with complex wall boundaries since the scheme alternates between two staggered grids every other time step. The method of Jiang et al. (1998) avoids this by providing a procedure to convert staggered schemes to a fixed nonstaggered grid.

The boundary conditions closely parallel the experimental set-up. At the lefthand boundary of the computational domain there is a supercritical inflow, where the thickness and velocity are prescribed. Along the sidewalls and the wedge a no-normalflux condition $h \boldsymbol{u} \cdot \boldsymbol{n}=0$ is applied, while at the outflow on the right the number of boundary conditions depends on whether the flow is sub- or supercritical (e.g. Weiyan 1992). For most simulations the outflow is supercritical and no boundary conditions need to be prescribed, but to simulate a strong shock the outflow conditions need to mirror the blocking procedure used in the experiments. Initially the flow is blocked completely by setting $m=h u=0$, so that a normal shock forms and propagates upslope. As the normal shock approaches the tip of the wedge the outflow is opened, at time $t_{o p e n}$. For a steady state to be established, the total inflow mass flux must balance the total outflow mass flux. Assuming that the inflow and outflow mass fluxes $m_{i}$ and $m_{o}$ are uniformly distributed across the chute, the appropriate downstream conditions are

$$
m_{o}= \begin{cases}0, & t<t_{\text {open }}, \\ W_{i} m_{i} / W_{o}, & t \geqslant t_{\text {open }},\end{cases}
$$

where $W_{i}$ and $W_{o}$ are the inflow and outflow chute widths, shown in figure 7. The boundary conditions are summarized in table 2 . The numerical method has been tested against the exact solutions in the absence of source terms and accurately reproduces the shock angles and the jumps in thickness and velocity given by (2.7), (2.6) and (2.10).

\section{Numerical results with non-zero source terms}

The classical oblique-shock solutions in $\S 2$ assume that the source terms are identically zero and hence that the states on either side of the shocks are constant. In real granular flows the source terms (1.5), (1.6) are never identically zero over the whole domain, and they necessarily induce gradients in the thickness and velocity that in turn modify the local upstream Froude number and the position of the shocks. In this section we use the numerical method described above to show that the full system of equations (1.2)-(1.6) can accurately predict the experimental position of 
the weak, strong and detached oblique shocks discussed in $\S 3$, and therefore that the source terms can explain the discrepancies from classical oblique-shock theory.

\subsection{Weak and strong oblique shocks}

Figure 8 shows the variation in the computed thickness, velocity and Froude number for the case of the experimental weak and strong shocks described in $\S 3$. The contoured domains are exactly equivalent to the overhead photographs shown in the right-hand panels of figure 6 . Since the slope induces a net acceleration of the flow, there is a steady increase in the velocity and a decrease in the thickness upstream of both shocks. The weak shock is almost straight and lies at an angle of approximately $28^{\circ}$ to the direction of flow, which compares well with the measured angle of $29^{\circ} \pm 1^{\circ}$ as well as the angle in the absence of source terms. Immediately after the shock the thickness jumps to 0.9 non-dimensional units, which is precisely the value measured in experiments, and then continues to increase slowly in height as the wedge is approached. This slow increase can also be seen in the perspective view of the experiments in figure 8. The velocity vectors lie almost parallel to the wedge after the shock and the velocity magnitude and hence the Froude number increase slowly with downstream distance along the wedge in response to the net acceleration provided by the source terms.

For exactly the same inflow conditions, careful control of the downstream gate enables a strong shock that lies across the entire chute to be triggered. To achieve this the second gate must be opened at time $t_{\text {open }}=27.0$ non-dimensional time units in the computation, which corresponds to 1.49 seconds. This compares favourably with the experimental opening time of $1.60 \pm 0.04$ seconds or $28.9 \pm 0.72$ non-dimensional time units. The numerical solutions are computed on the complete domain shown in figure 7 but only a small section of the solution, which corresponds to the experimental domain in figure 6 , is illustrated. When viewed over the whole domain the strong shock is almost linear and inclined at an angle of approximately $77^{\circ}$ to the direction of flow, but close to the wedge there are some significant variations. These arise because the outflow conditions have an upstream influence on the position of the shock, and, close to the wedge, the wall condition produces a shock angle slightly different from the constant-outflow condition along the rest of the domain. The computed strongshock angle is very close to the experimental value of $78^{\circ} \pm 1^{\circ}$, which is significantly better than the value of $86.15^{\circ}$ predicted by the theory in the absence of source terms. The jump in thickness to 2.0 non-dimensional units immediately after the shock is once again exactly the same as that measured in the experiments. However, the thickness continues to increases significantly in height as the second blocking gate is approached, and the pressure gradients that this induces are sufficient to continue to slow the flow down, even though the slope naturally provides a net acceleration. The thickness increase after the shock is very significant and, if strong shocks are to be used as a control measure, then the dam height must also increase with downstream distance.

\subsection{Energy dissipation}

If strong shocks can be induced by dams in the field then they have the potential to provide significantly greater protection from hazardous geophysical flows than deflecting dams that induce weak shocks, because they dissipate far larger amounts of energy. To make this explicit, the depth-integrated energy balance (e.g. Johnson 1997) necessarily implies that to leading order the energy jump $\mathscr{E}$ across a 

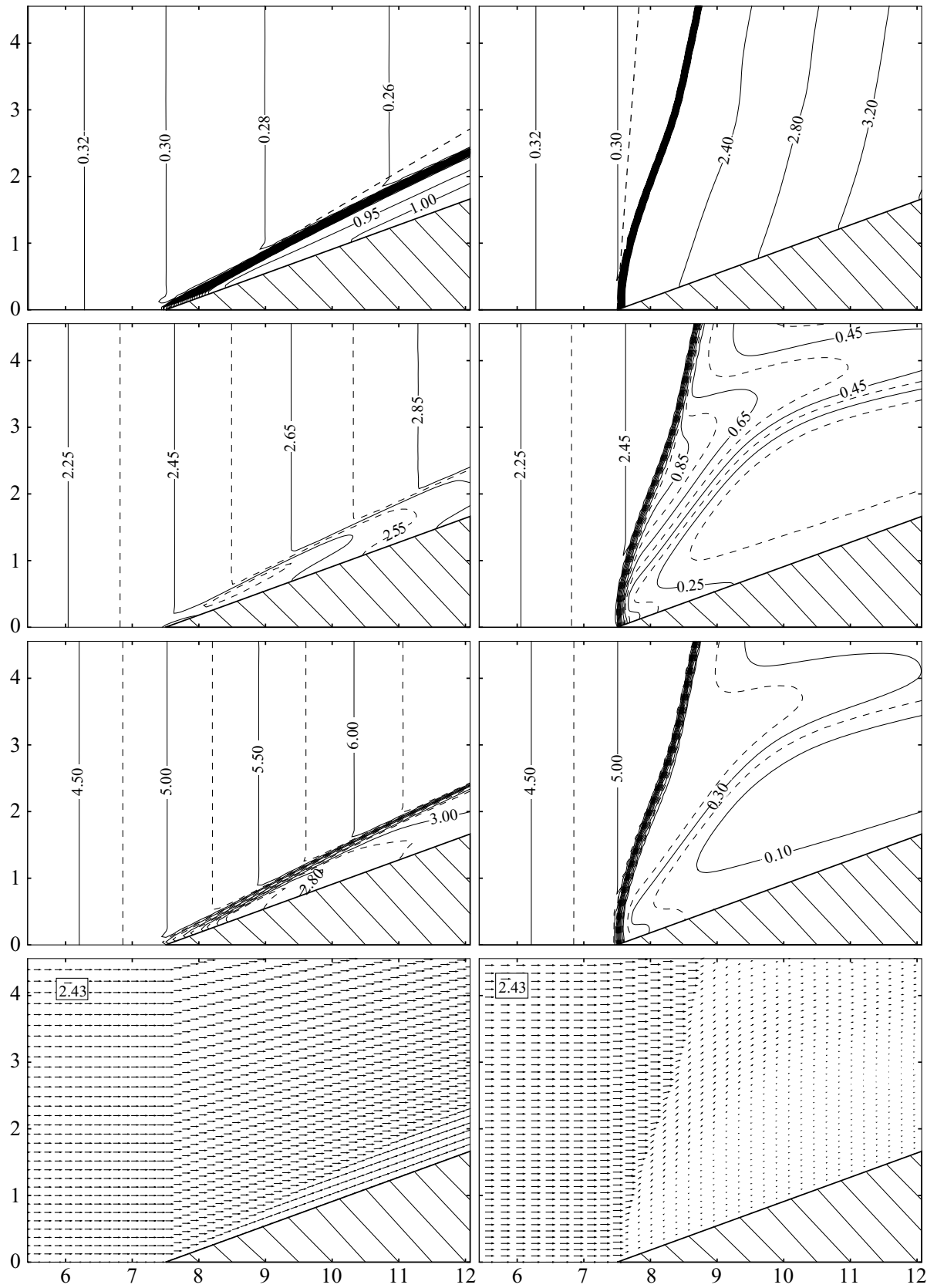

FIGURE 8 . The top three pairs of panels show non-dimensional contour plots of the flow thickness $h$, the velocity magnitude $|\boldsymbol{u}|$ and the Froude number $F r$ for a weak shock (left) and a strong shock (right) with source terms. The bottom pair of panels shows the corresponding velocity vectors. The $x$-axis and downslope direction are along the abscissa and the cross-slope $y$-axis lies along the ordinate. The tip of the wedge lies at $x=7.5$ and is inclined an angle of $20^{\circ}$ to the downstream direction. In both cases the local Froude number at the wedge, $\mathrm{Fr}$ equals 5 , and the local thickness and velocity are equal to $h=0.3$ and $u=2.43$, respectively. The chute is inclined at $\zeta=38^{\circ}$ to the horizontal and the basal angle of friction $\delta=23^{\circ}$. The dashed lines in the top pair of panels indicate the position of the weak (left) and strong (right) shocks in the absence of source terms. 
two-dimensional shock is

$$
\mathscr{E}=\llbracket(\boldsymbol{u} \cdot \boldsymbol{n})\left(\frac{1}{2} h|\boldsymbol{u}|^{2}+\varepsilon h^{2} \cos \zeta\right) \rrbracket-v_{n} \llbracket \frac{1}{2} h|\boldsymbol{u}|^{2}+\frac{1}{2} \varepsilon h \cos \zeta \rrbracket .
$$

It follows that for a steady oblique shock the energy loss is given by

$$
\mathscr{E}=\llbracket(\boldsymbol{u} \cdot \boldsymbol{n})\left(\frac{1}{2} h|\boldsymbol{u}|^{2}+\varepsilon h^{2} \cos \zeta\right) \rrbracket>0 .
$$

The steady-mass jump condition (1.8) provides a natural definition for the normal mass flux $M_{n}$ across a steady shock, i.e.

$$
M_{n}=h_{1}\left(\boldsymbol{u}_{1} \cdot \boldsymbol{n}\right)=h_{2}\left(\boldsymbol{u}_{2} \cdot \boldsymbol{n}\right),
$$

where the subscript 1 indicates a position just upstream of the shock and the subscript 2 a position just downstream of it. Using these definitions and that $\llbracket|\boldsymbol{u}|^{2} \rrbracket=$ $\left(\boldsymbol{u}_{1}+\boldsymbol{u}_{2}\right) \cdot\left(\boldsymbol{u}_{1}-\boldsymbol{u}_{2}\right),(5.2)$ can be rewritten as

$$
\mathscr{E}=\frac{1}{2}\left(\boldsymbol{u}_{1}+\boldsymbol{u}_{2}\right) \cdot \llbracket h \boldsymbol{u}(\boldsymbol{u} \cdot \boldsymbol{n}) \rrbracket+\varepsilon M_{n} \llbracket h \rrbracket \cos \zeta .
$$

Since the steady-momentum jump condition (1.9) implies

$$
\llbracket h \boldsymbol{u}(\boldsymbol{u} \cdot \boldsymbol{n}) \rrbracket=-\frac{1}{2} \varepsilon \cos \zeta \llbracket h^{2} \rrbracket \boldsymbol{n},
$$

the energy loss across a steady oblique shock reduces to

$$
\mathscr{E}=\frac{M_{n} \varepsilon \cos \zeta}{4 h_{1} h_{2}}\left(h_{2}-h_{1}\right)^{3} .
$$

This expression is similar to the energy loss across a hydraulic jump (e.g. Stoker 1957; Johnson 1997) since there is a dependence on the cube of the thickness difference between the two sides, but it also depends on the slope inclination angle and the normal mass flux $M_{n}$, defined in (5.3). Strong shocks dissipate much more energy than weak shocks, for two reasons; firstly there is a much larger jump in thickness and secondly the incoming velocity $\boldsymbol{u}_{1}$ and the normal $\boldsymbol{n}$ are closely aligned, so there is a much greater mass flux $M_{n}$ across the shock. As a result, for the experiments in $\S 3$, the strong shock dissipates over 20 times more energy than the weak shock. The only disadvantages of the strong-shock set-up are that the wedge has to be significantly higher to prevent overtopping and the downstream conditions have to be carefully controlled.

\subsection{Detached oblique shocks}

When the deflection angle of the wedge is increased to $\theta=44^{\circ}$ and the incoming Froude number is decreased to $F r_{1}=4$, the shock detaches from the wedge tip to produce a detached oblique shock, as predicted by the exact solution in figure 3 . The left-hand panels in figure 9 show the computed solution in the absence of source terms. The shock initially starts off as a normal shock about 0.5 non-dimensional units upstream of the tip and then slowly bends towards the wedge with increasing downstream distance. Even in the absence of source terms and with a uniform upstream state the downstream thickness and velocity are not constant. The initial normal shock produces the largest jump in thickness and decrease with velocity, and, as one moves along the wedge, the thickness slowly decreases and the velocity increases. The right-hand panels of figure 9 show the effect of including source terms and are directly comparable with the bottom right overhead experimental photo in figure 6. The shock is once again detached, so the curve in figure 3 gives useful approximations for the detachment angle even with the presence of source terms. 

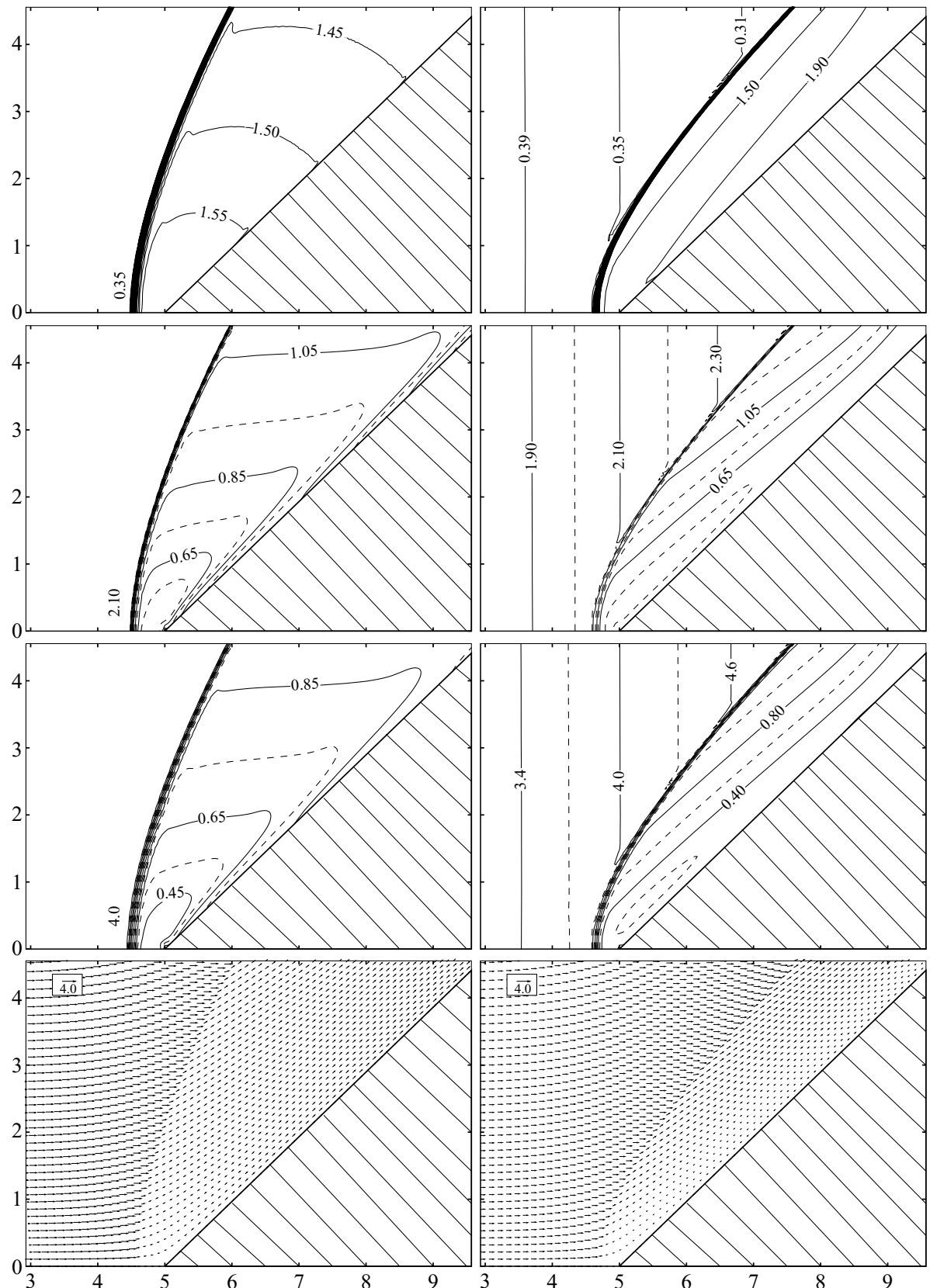

FIGURE 9. The top three pairs of panels show non-dimensional contour plots of the flow thickness $h$, the velocity magnitude $|\boldsymbol{u}|$ and the Froude number $F r$ for a detached oblique shock in the absence of source terms (left) and with source terms (right). The bottom pair of panels show the corresponding velocity vectors. The $x$-axis and downslope direction are along the abscissa and the cross-slope $y$-axis is along the ordinate. The tip of the wedge lies at $x=5$ and is inclined at $\theta=44^{\circ}$ to the downstream direction. The undisturbed incoming flow has a local Froude number $F r=4$ at $x=5$ and the thickness and velocity are $h=0.35$ and $u=2.10$, respectively. The chute is inclined at $\zeta=38^{\circ}$ to the horizontal and the basal angle of friction $\delta=23^{\circ}$ in the plots on the right. 
The computed shock detaches about 0.35 non-dimensional units above the tip, which compares very well with the observed length and lies slightly downstream of the position in the absence of source terms. The key effect is to bend the shock around towards the wedge much more rapidly, so that it lies much closer to it. This is in excellent agreement with the experiments. As one moves normally from the shock towards the wedge the thickness slowly increases, which can also be seen in the perspective profile in the bottom left photograph in figure 6. Similarly, as one moves towards the wedge the flow velocity decreases. Detached oblique shocks produce large thickness jumps and big deflection angles, so they dissipate significant amounts of energy along their length. They may therefore be very useful for protective measures as they do not require a complicated triggering mechanism to allow them to form.

\subsection{Accelerative and decelerative slopes}

The classical shock-deflection-angle curves provide useful approximations for the shock angles in most situations. However, it is also possible to produce significant deviations from them at low Froude numbers. Figure 10 shows two sets of weakshock solutions for inflow Froude number $F r_{1}=4.15$, non-dimensional thickness $h_{1}=0.1$ and basal angle of friction $\delta=23^{\circ}$. The panels on the left correspond to a slightly decelerative slope with $\zeta=21^{\circ}$, while the panels on the right correspond to an accelerative slope with $\zeta=30^{\circ}$. The wedge deflection angle $\theta$ is $20^{\circ}$ in both cases, so the classical shock-deflection-angle relation (2.7) predicts a common shock angle of $\beta=33.39^{\circ}$. On the decelerative slope the shock slowly bends away from the classical angle to approximately $36^{\circ}$ by the end of the domain, while on the accelerative slope it bends towards the wedge and attains an angle of $27.5^{\circ}$. For a weak shock, much of this deviation can be explained by the variation in the incoming Froude number, $F r_{1}$, along its length. On the decelerative slope the Froude number decreases from 4.15 to about 3.5 by the end of the chute. Assuming that the flow is parallel to the wedge after the shock, the classical shock-deflection-angle relation (2.7) predicts that the shock angle should continuously increase from $33.39^{\circ}$ to $36.59^{\circ}$. An approximation for the weak-shock position may therefore be made by solving the ordinary differential equation

$$
\frac{\mathrm{d} y}{\mathrm{~d} x}=\tan \beta\left(F r_{1}(x)\right),
$$

from the tip of the wedge using the actual variation in the Froude number with $x$ to calculate the changes in the local shock angle $\beta$ using (2.7). This shows that the shock is actually slightly concave, bending away from the wedge with increasing downstream distance as observed in the computations. Similarly, when the shock is on an accelerative slope the Froude number increases from 4.15 to about 6.5, which produces a corresponding decrease in angle from $\beta=33.39^{\circ}$ to $27.87^{\circ}$. Solving (5.6) in this case results in a slightly convex curve that accounts for about half the observed decrease in angle. The remaining difference is due to variations in the velocity on the downstream side of the shock, so that it is not exactly parallel to the wedge immediately after it, as assumed in the classical result (2.7).

\section{Conclusions}

In this paper we have shown that weak, strong and detached oblique shocks may be generated when granular free-surface flows are deflected by a wedge or obstacle. The classical hydraulic shock-deflection-angle relation (2.7) provides a good first 

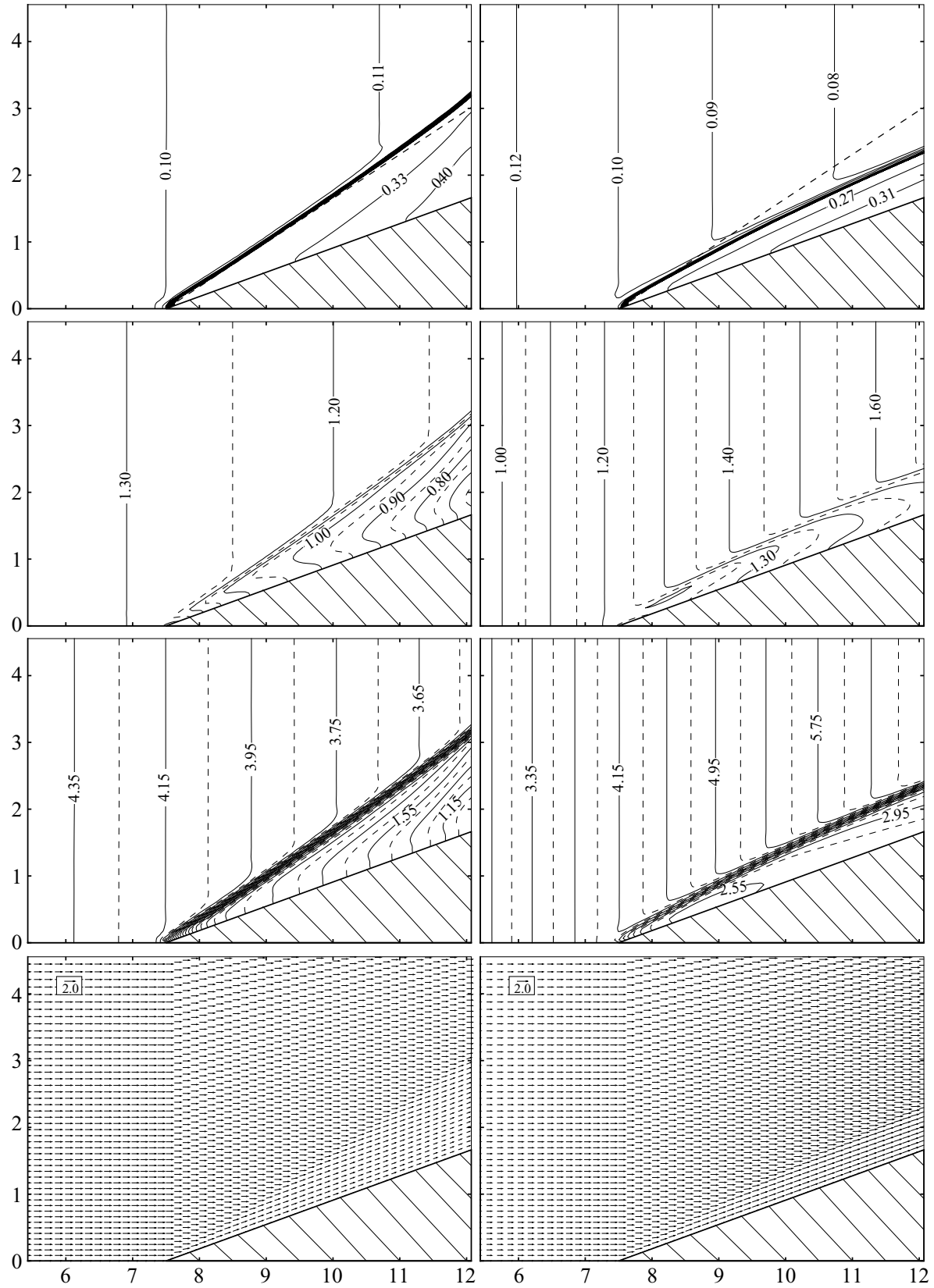

FIGURE 10. The top three pairs of panels show non-dimensional contour plots of the flow thickness $h$, the velocity magnitude $|\boldsymbol{u}|$ and the Froude number $F r$ for a weak shock on a decelerative slope with $\zeta=21^{\circ}$ (left) and on an accelerative slope with $\zeta=30^{\circ}$ (right). The bottom pair of panels show the corresponding velocity vectors. The $x$-axis and downslope direction are along the abscissa and the cross-slope $y$-axis lies along the ordinate. The tip of the wedge lies at $x=7.5$ and is inclined at an angle of $20^{\circ}$ to the downstream direction, and the basal angle of friction $\delta$ equals $23^{\circ}$. The local Froude number at the wedge, $\mathrm{Fr}$ equals 4.15 , and the thickness $h$ is 0.1 . This implies that the velocity $u=1.27$ for the $21^{\circ}$ slope and 1.22 for $30^{\circ}$. The dashed lines in the top pair of panels indicate the position of the weak shock in the absence of source terms and lies at $33.39^{\circ}$ for both cases. 
approximation for the angles of the observed weak and strong shocks. Weak shocks form naturally during flows past wedges, provided that the Froude number is high enough and the deflection angle is not too large, and produce a narrow region close to the wedge with increased flow thickness and decreased velocity. The generation of strong shocks, however, requires careful control of the downstream conditions. Strong shocks produce much larger increases in the thickness and decreases in the velocity, and the shock extends across the chute. Equation (5.5) shows that both these effects imply that strong shocks dissipate much more energy than weak shocks and they may therefore provide a useful defence mechanism against large-scale hazardous geophysical flows.

A new exact solution (2.9) for the detachment Froude number $F r_{d}$ provides a useful criterion to predict whether the flow will form a detached oblique shock, even on slopes where the source terms play a significant role. Detached shocks also dissipate large amounts of energy, because they necessarily involve large shock angles and jumps in thickness. As the key design control is simply the wedge angle $\theta$, these may provide a more easily realizable defensive strategy although they do not provide as much dissipation as strong shocks.

The effects of gravitational acceleration and Coulomb basal friction, which enter primarily through the source terms on the right-hand side of (1.3) and (1.4), have been investigated using shock-capturing numerical methods. These show that the full system of governing equations (1.2)-(1.6) provides a very accurate model for computing the actual positions of weak, strong and detached shocks and the variations in the flow thickness and velocity. The simulations indicate that the largest deviations from the classical shock-deflection angle (2.7) occur at low incoming Froude numbers on strongly accelerative or decelerative slopes. On accelerative slopes weak shocks bend towards the wedge, while on decelerative slopes they bend away from it. An approximation for the modified position of a weak shock is obtained by solving (5.6) as a function of the variation in the local upstream Froude number with downstream distance. In both cases, the thickness of the downstream flow increases with increasing downstream distance along the dam. This is contrary to current design criteria for defensive dams (e.g. Sigurdsson, Tomasson \& Sandersen 1998), which suggest lower heights further down the run-out zone, but is in accordance with observed snowavalanche deposits along the dam at Flateyri in Northwestern Iceland (Jóhannesson 2001; Cui et al. 2007).

This research was generously supported by the EPSRC (GR/R753-4/01), NERC (NER/A/S/2003/00439) and the Manchester Centre for Nonlinear Dynamics. Nico Gray also gratefully acknowledges the support of an EPSRC Advanced Research Fellowship (GR/S50052/01 and GR/S50069/01).

\section{REFERENCES}

Abramowitz, M. \& Stegun, I. 1970 Handbook of Mathematical Functions, 9th Edn. Dover.

Amarouchene, Y. \& Kellay, H. 2006 Speed of sound from shock fronts in granular flows. Phys. Fluids 18, 031707.

Ames Research Staff 1953 Equations, tables and charts for compressible flow. NACA Rep. 1135.

Anderson, J. D. 1982 Modern Compressible Flow. McGraw-Hill.

Aksel, M. H. \& Eralp, O. C. 1994 Gas Dynamics. Prentice Hall.

Brater, E. F., King, H. F., Lindell, J. E. \& Wei, C. Y. 1996 Handbook of Hydraulics, 7th Ed. McGraw-Hill. 
Brennan, C. E., Sieck, K. \& Paslaski J. 1983 Hydraulic jumps in granular material flow. Powder Tech. 35 31-37.

Chadwick, P. 1976 Continuum Mechanics. Concise Theory and Problems. George Allen \& Unwin (republished Dover 1999).

Cui, X., Gray, J. M. N. T. \& Jóhannesson, T. 2007 Delecting dams and the formation of oblique shocks in snow avalanches at Flateyri, Iceland. J. Geophys. Res. (in press).

Denlinger, R. P. \& Iverson, R. M. 2001 Flow of variably fluidized granular masses across threedimensional terrain 2. Numerical predictions and experimental tests. J. Geophys. Res. B1, 553-566.

Gray, J. M. N. T. \& Hutter, K. 1997 Pattern formation in granular avalanches. Continuum Mech. Thermodyn. 9, 341-345.

Gray, J. M. N. T. \& Hutter, K. 1998 Physik granularer Lawinen. Physikalische Blatter 54(1), $37-43$.

Gray, J. M. N. T., Irmer, A., TaI, Y. C. \& Hutter K. 1999a Plane and oblique Shocks in shallow granular flows. In 22nd Intl. Symp. on Shock Waves, Imperial College, London, UK, July 18-23, 1999. Paper 4550, 1447-1452, Bound Proceedings ISBN 0854327118, CD-Rom ISBN 0845327061.

Gray, J. M. N. T. \& TAI, Y. C. 1998 Particle size segregation, granular shocks and stratification patterns. In Physics of Dry Granular Media (ed. H. J. Herrmann, J. P. Hovi \& S. P. Luding), Nato ASI Series E: Applied Sciences, vol. 350, pp. 697-702.

Gray, J. M. N. T., TaI, Y. C. \& Noelle, S. 2003 Shock waves, dead-zones and particle-free regions in rapid granular free-surface flows. J. Fluid Mech. 491, 161-181.

Gray, J. M. N. T., Wieland, M. \& Hutter, K. $1999 b$ Free surface flow of cohesionless granular avalanches over complex basal topography. Proc. R. Soc. Lond. A 455, 1841-1874.

Grigorian, S. S., Eglit, M. E. \& Iakimov, IU. L. 1967 New state and solution of the problem of the motion of snow avalance. Snow, Avalanches \& Glaciers. Tr. Vysokogornogo Geofizich Inst. 12, 104-113.

Hákonardóttir, K. M. \& Hogg, A. J. 2005 Oblique shocks in rapid granular flows. Phys. Fluids 17, 0077101.

Heil, P., Rericha, E. C., Goldman, D. I. \& Swinney, H. L. 2004 Mach cone in a shallow granular fluid. Phys. Rev. E 70, 060301.

IPPEN, A.T. 1949 Mechanics of supercritical flow. ASCE. 116, 268-295.

Irmer, A., Schaefer, M., Gray, J. M. N. T. \& Voinovich, P. 1999. An adaptive unstructured solver for granular flows. In 22nd Intl Symp. on Shock Waves, Imperial College, London, UK, July 18-23, 1999. Paper 4610, 689-693, Bound Proceedings ISBN 0854327118, CD-Rom ISBN 0845327061.

IVErson, R. M. 1997 The physics of debris-flows. Rev. Geophys. 35, 245-296.

Jiang, G.-S., Levy, D., Lin, C.-T., Osher, S. \& Tadmor, E. 1998 High-resolution nonoscillatory central schemes with nonstaggered grids for hyperbolic conservation laws. SIAM J. Numer. Anal. 35, 2147-2168.

JiANG, G. \& TADMOR, E. 1998 Non-oscillatory central schemes for multidimensional hyperbolic conservation laws. SIAM J. Sci. Comput. 19(6), 1892-1917.

JóHANNESSON, T. 2001 Run-up of two avalanches on the deflecting dams at Flateyri, Northwest Iceland. Ann. Glaciol. 32, 2147-2168

Johnson, R. S. 1997 A modern introduction to the mathematical theory of water waves. Cambridge University Press.

Kulikovskil, A. G. \& Eglit, M. E. 1973 Two-dimensional problem of the motion of a snow avalanche along a slope with smoothly changing properties. J. Appl. Maths Mech. 37(5), 792-803.

Lie, K. A. \& NoELle, S. 2003 An improved quadrature rule for the flux-computation in highresolution nonoscillatory central difference schemes for systems of conservation laws in multidimensions. J. Sci. Comput. 18, 69-81.

Nessyahu, H. \& TAdmoR, E. 1990 Non-oscillatory central differencing for hyperbolic conservation laws. J. Comput. Phys. 87, 408-463.

Pouliquen, O. 1999 Scaling laws in granular flows down rough inclined planes. Phys. Fluids 11(3), $542-548$. 
Rericha, E. C., Bizon, C., Shattuck, M. \& Swinney, H. 2002 Shocks in supersonic sand. Phys. Rev. Lett. 88, 014302.

Rouse, H. 1938 Fluid Mechanics for Hydraulic Engineers. McGraw-Hill.

Rouse, H. 1949 Engineering Hydraulics. Wiley.

SAAD, M. A. 1993 Compressible Fluid Flow. Prentice-Hall.

SAvaGE, S. B. 1979 Gravity flow of cohesionless granular materials in chutes and channels. J. Fluid Mech. 92, 53-96.

Savage, S. B. \& Hutter, K. 1989 The motion of a finite mass of granular material down a rough incline. J. Fluid Mech. 199, 177-215.

Sigurdsson, F. Tomasson, G. G. \& Sandersen F. 1998 Avalanche defences for Flateyri, Iceland. From hazard evaluation to construction of defences. Norwegian Geotech. Inst. Rep. 203.

Shinbrot, T. \& Muzzio, F. J. 2000 Nonequilibrium patterns in granular mixing and segregation. Physics Today March, 25-30.

Sparks, R. S. J., Barclay, J., Calder, E. S. et al. 2002 Generation of a debris avalanche and violent pyroclastic density current on 26 December (Boxing Day) 1997 at Soufriere Hills Volcano, Monserrat. In The eruption of Soufriere Hills Volcano, Monserrat, from 1995 to 1999 (ed. Druitt \& Kokelaar). Geological Society, London, Memoirs, vol. 21, pp. 409-434.

Spurling, R. J., Davidson, J. F. \& ScOTT, D. M. 2001 The transient response of granular flows in an inclined rotating cylinder. Trans. Inst. Chem. Engrs 79(A), 51-61.

StOKeR, J. J. 1957 Water Waves. Interscience.

Vallance, J. W. 2000 Lahars. In Encyclopedia of Volcanoes, pp. 601-616. Academic.

WeIYAN, T. 1992 Shallow Water Hydrodynamics. Elsevier.

Wieland, M., Gray, J. M. N. T. \& HutTer, K. 1999 Channelized free surface flow of cohesionless granular avalanches in a chute with shallow lateral curvature. J. Fluid Mech. 392, 73-100. 